\title{
Thermoneutral mice heat up research
}

Though it varies from person to person, the average human's thermoneutral point is around $22^{\circ} \mathrm{C}$. This tends to also be the temperature of the average vivarium. The thermoneutral point for a mouse, however, is about $30^{\circ} \mathrm{C}$. Researchers and technicians who work with mice may be comfortable, but the animals themselves are likely cold. Imagine yourself outside on a $50^{\circ} \mathrm{F}$ day without a jacket, says Daniel Giles, a former $\mathrm{PhD}$ student in the lab of immunobiologist Senad Divanovic at Cincinnati Children's Hospital, and that's the daily experience of the average lab mouse.

One area of study in Divanovic's lab is non-alcoholic fatty liver disease (NAFLD). Linked to growing waistlines, this chronic condition is estimated to affect about a third of Americans, including an increasing number of adolescents. Untreated, the liver becomes inflamed. Prolonged damage can lead to cirrhosis or even hepatocellular carcinoma. Weight loss and controlling other risk factors like high cholesterol and type II diabetes can help, but unchecked progression currently culminates in the need for a liver transplant. Millions of Americans are at risk, but there aren't millions of available livers to go around.

As NAFLD cases rise, researchers and clinicians are searching for more viable treatment options than weight loss or organ transplants. Basic and preclinical research relies heavily on mice, but NAFLD has proved hard to model. High-fat diets can induce obesity to an extent, but the animals generally don't develop the inflamed livers or hepatic fibrosis observed in people. Knockout lines capture some human aspects, but not the complete picture. And even though the condition afflicts human sexes equally, obese female mice can be hard to come by without drastic interventions, like ovariectomies or genetic manipulations.

Obesity is a complicated condition, but one detail that is clear, says Divanovic, is that inflammation has a role to play in its ill

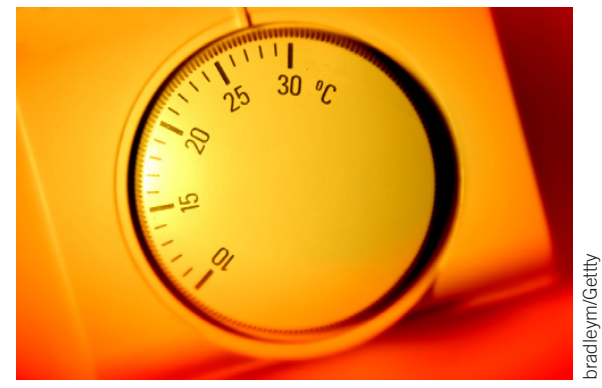

Cold stress appears to dampen immune responses in general, with implications for obesity and NAFLD as a result.

The microbiome may also be at playlikely adding on to the immune effects-in a way that mirrors what happens in humans with fatty livers. Bacteroidetes species increase at thermoneutrality while another group, the Firmicutes, decrease. Knocking out Bacteroidetes with antibiotics kept the mice from developing signs of NAFLD.

Although the study did not reveal effects on the body. With previous research linking body temperature to immune and metabolic responses in mice, Giles and Divanovic decided to crank up the thermostat (Nat. Med. 2017; doi:10.1038/ nm.4346).

The team took advantage of a specially built room in Cincinnati Children's animal facility that could be kept much warmer than normal. They compared obesity and signs of NAFLD in wild-type mice with the conventional approach of using standard chow versus a high-fat diet, but under two different housing temperatures: $22^{\circ} \mathrm{C}$, the vivarium standard, and $30^{\circ} \mathrm{C}$, murine thermoneutral.

Their results suggest temperature matters. Over the course of 24 weeks, warm C57BL/6 mice exhibited similar weight gain, but had heavier livers, higher triglyceride levels and an increased expression of fibrosis markers compared to their colder counterparts. Notably, female mice housed at thermoneutrality also developed severe obesity and hepatocellular damage. Another mouse strain used in NAFLD modeling, the AKR mouse, confirmed the findings and even had observable signs of liver fibrosis.

Looking under the surface, the differences seem to be linked to an upregulated immune system at thermoneutrality. Using in vivo cytokine capture analysis, the researchers saw a baseline increase in cytokine levels-both pro-inflammatory and anti-inflammatory-in healthy, $30^{\circ} \mathrm{C}$ mice. the exact mechanism that exacerbates NAFLD pathogenesis at thermoneutrality, Divanovic and Giles think they have an important tool to create more relevant mouse models for interrogation in the future. Divanovic's lab is especially excited to follow up with female mice. With evidence in humans that a child's weight can be influenced by that of their parents, the ability to test both paternal and maternal obesity opens new avenues of research.

For the time being, Giles has turned his attention to inflammatory bowel disease in people and bioinformatics at the La Jolla Institute for Allergy and Immunology. "Right now, I'm trying to learn how to better analyze human data, make hypotheses, and design mouse experiments based on what we see in humans," he says, hoping to someday return to obesity and thermoneutral mice.

These results aren't the first to suggest the importance of thermoneutral housing in laboratory mice - the paper joins others on atherosclerosis, bacterial and viral infections, tumor clearance, and graft versus host disease in the literature-nor is it likely to be the last. Sweltering vivariums (to people) probably won't replace climatecontrolled ones entirely, but vendors are responding with better temperature control within the racks and individual cages.

Warmer mice may be just what the doctor ordered.

Ellen P. Neff 